\title{
Case report: Transfusion-related acute lung injury (TRALI) - A clear and present danger
}

\author{
[Présentation de cas : Lésion pulmonaire aiguë associée à la transfusion (TRALI) \\ - Un danger net et présent]
}

Yulia Lin MD, ${ }^{*}$ Nisha Kanani MD, † Finola Naughton MD, † Jacob Pendergrast MD, ${ }^{\star}$ Keyvan Karkouti MD†‡

Purpose: To describe a case of transfusion-related acute lung injury (TRALI) after platelet transfusion immediately following cardiac surgery, and to review the clinical features, pathophysiology, management, and morbidity and mortality associated with such an event.

Clinical features: A 62-yr-old man was transferred to our centre for urgent coronary artery bypass grafting in the setting of recent anti-platelet medication use. Soon after surgery he received platelet transfusions despite having only moderate blood loss. Shortly following the platelet transfusion, he suffered acute hypoxic and hypotensive decompensation requiring nitric oxide therapy, inotropic support, and prolonged need for mechanical ventilation. The patient was eventually discharged from the intensive care unit nine days following the event. The diagnosis of TRALI was made by clinical and radiographic criteria.

Conclusion: Transfusion-related acute lung injury is now the leading cause of transfusion-related fatalities. Early diagnosis of TRALI is important and these reactions should be reported to the blood transfusion service so that appropriate action can be taken to prevent future morbidity and mortality in other patients. To reduce serious transfusion reactions, inappropriate transfusions must be minimized and the decision to transfuse blood products should be taken with care.

CAN J ANESTH 2007 / 54:12/pp 1011-1016

Objectif : Décrire le cas d'une lésion pulmonaire aiguë associée à la transfusion (TRALI) après la transfusion de plaquettes immédiatement après une chirurgie cardiaque, et faire un compte-rendu des éléments cliniques, de la physiopathologie, de la prise en charge, de la morbidité et de la mortalité associés à un tel cas.
Éléments cliniques: Un homme de 62 ans a été transféré à notre centre pour un pontage aortocoronarien urgent dans le cadre de l'utilisation récente d'une médication anti-plaquettaire. Peu de temps après la chirurgie, le patient a reçu une transfusion de plaquettes malgré une perte sanguine très modérée. Peu après la transfusion de plaquettes, il est devenu hypoxique et hypotendu, ce qui a nécessité un traitement à l'oxyde nitrique, un soutien inotrope et le besoin de ventilation mécanique prolongée. Finalement, le patient a eu son congé des soins intensifs neuf jours après l'opération. Le diagnostic d'une TRALI a été fait sur la base de critères cliniques et radiographiques.

Conclusion : Aujourd'hui, les lésions pulmonaires aiguës associées à la transfusion sont la cause principale de décès associés à la transfusion. Le diagnostic précoce d'une TRALI est important et ces réactions devraient être rapportées au service de transfusion sanguine afin que des mesures appropriées puissent être prises pour éviter la morbidité et la mortalité d'autres patients dans le futur. Pour réduire les réactions sérieuses à la transfusion, les transfusions inadéquates doivent être minimisées et la décision de transfuser des produits sanguins prise avec soin.

From the Departments of Laboratory Medicine and Pathobiology, ${ }^{*}$ Anesthesia, $\uparrow$ and Health Policy, Management, and Evaluation, $\ddagger$ University of Toronto, Toronto, Ontario, Canada.

Address correspondence to: Dr. Keyvan Karkouti, Toronto General Hospital, University Health Network, Department of Anesthesia, 3 Eaton North, 200 Elizabeth Street, Toronto, Ontario M5G 2C4, Canada. Phone: 416-340-5164; Fax: 416-340-3698; E-mail: keyvan. karkouti@uhn.on.ca

Funding and conflicts of interest: K. Karkouti is supported in part by the Canadian Anesthesiologists' Society Research Foundation. Accepted for publication August 23, 2007.

Revision accepted September September 20, 2007. 
recent clopidogrel use, despite having only moderate blood loss, and who developed near fatal transfusion-related acute lung injury (TRALI) caused by the platelet transfusion. The patient provided informed consent for publication of this report detailing his perioperative course.

\section{Clinical report}

A 62-yr-old-man (weighing $95 \mathrm{~kg}$ ) with known coronary artery disease (two historically remote myocardial infarctions and several coronary artery stents), hypertension, and sleep apnea was transferred to our centre with unstable angina for urgent coronary artery re-vascularization. Coronary catheterization revealed greater than $70 \%$ stenosis of the left main, left anterior descending, and circumflex coronary arteries, and mild (grade II) left ventricular dysfunction. His medications at the time of admission included clopidogrel and acetyl-salicylic acid, as well as metoprolol, simvastatin, ramipril, amlodipine, indapamide, and oxazepam.

Two days following admission, he underwent coronary artery bypass grafting (saphenous vein bypass to an obtuse marginal and a diagonal artery, and a left internal thoracic artery bypass to the left anterior descending artery). Before initiation of cardiopulmonary bypass, he received tranexamic acid $100 \mathrm{mg} \cdot \mathrm{kg}^{-1}$ iv. Cardiopulmonary bypass time was $101 \mathrm{~min}$, with a cross clamp time of $84 \mathrm{~min}$. Before surgical closure, activated clotting time was normalized and hemostasis was deemed satisfactory. Given the history of recent anti-platelet drug use, he was given DDAVP 20 ug iv after discontinuation of cardiopulmonary bypass. He required no intraoperative blood transfusions. Laboratory tests after heparin reversal were: hemoglobin $119 \mathrm{~g} \cdot \mathrm{L}^{-1}$, platelet count $154 \times 10^{9} \cdot \mathrm{L}^{-1}$, international normalized ratio 1.5 , activated partial thromboplastin time $24 \mathrm{sec}$, and fibrinogen $2.23 \mathrm{~g} \cdot \mathrm{L}^{-1}$.

Upon transfer to the intensive care unit, the patient was stable, requiring only intravenous propofol infusion for sedation and nitroglycerin infusion for control of elevated blood pressure. Fifty minutes after admission, he had lost $70 \mathrm{~mL}$ of blood via the chest drains. In response, and influenced by his recent anti-platelet drug use, five units of platelets were transfused over $15 \mathrm{~min}$. Twenty-five minutes later, the patient suffered an acute hypoxic (trough $\mathrm{SpO}_{2}=65 \%$ ), hypotensive (trough blood pressure $=55 / 35 \mathrm{mmHg}$ ) episode. Copious frothy pulmonary edema exuded from the endotracheal tube. Bilateral rales were heard on chest auscultation. There was no evidence of rash, angioedema or bronchospasm to suggest an allergic reaction, and no evidence of cardiac arrhythmia, ischemia or hemorrhage to account for the decrease in blood

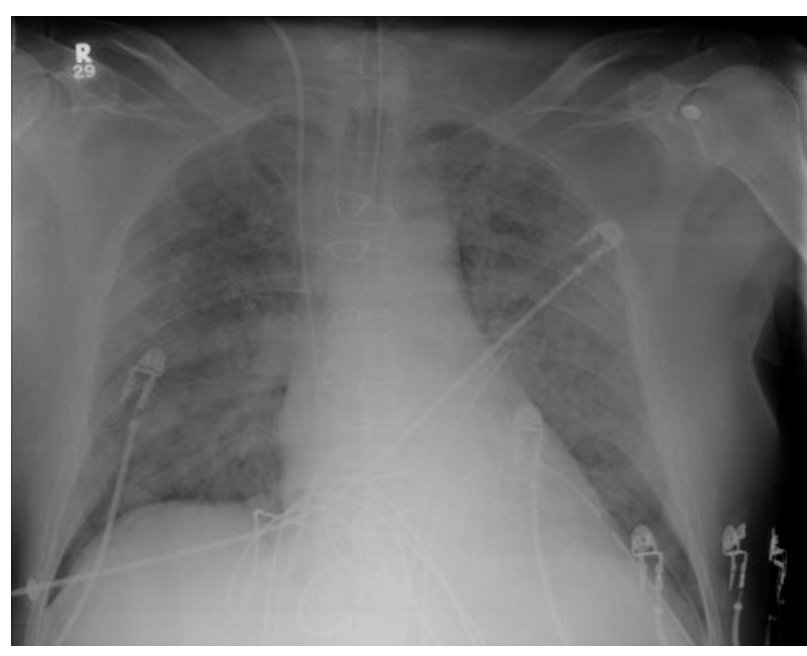

FIGURE 1 Portable antero-posterior chest $x$-ray 45 min after transfusion of five units of platelets.

pressure. There was no evidence of circulatory overload with central venous pressure measured at 10 $\mathrm{mmHg}$ and transesophageal echocardiography showed normally functioning, but under-filled left and right ventricles.

The chest $x$-ray, which had shown clear lung fields the day before, now revealed bilateral, diffuse airspace consolidation (Figure 1). The patient remained afebrile and laboratory investigations obtained immediately following symptom onset (including blood cultures and hemolytic indices) were unremarkable. On the basis of these clinical and radiolographic findings, a diagnosis of TRALI was made.

The patient was under continuous hemodynamic monitoring postoperatively including measurements obtained by a pulmonary artery (PA) catheter (Figure 2 ). Notably, the first manifestation of the reaction was an increase in PA pressure occurring $15 \mathrm{~min}$ before the onset of hypoxemia. As the PA pressure peaked at $53 / 26 \mathrm{mmHg}$, the patient's arterial oxygen saturation declined precipitously to $67 \%$ accompanied by a fall in systemic arterial blood pressure. As the mean arterial pressure fell below $60 \mathrm{mmHg}$, the mean PA pressure fell abruptly to $10 \mathrm{mmHg}$. During this time, his cardiac index was $2.1 \mathrm{~L} \cdot \mathrm{min}^{-1} \cdot \mathrm{m}^{-2}$, with a calculated systemic vascular resistance of 799 dynes $\cdot \mathrm{sec}^{-1} \cdot \mathrm{cm}^{-5}$. A transesophageal echocardiogram performed at the bedside revealed normal left and right ventricular function, but both chambers were under filled.

The patient's hypoxemia was managed by increasing $\mathrm{F}_{\mathrm{I}} \mathrm{O}_{2}$ to $100 \%$, increasing the positive end-expiratory pressure to $15 \mathrm{mmHg}$, and initiating $20 \mathrm{ppm}$ of 

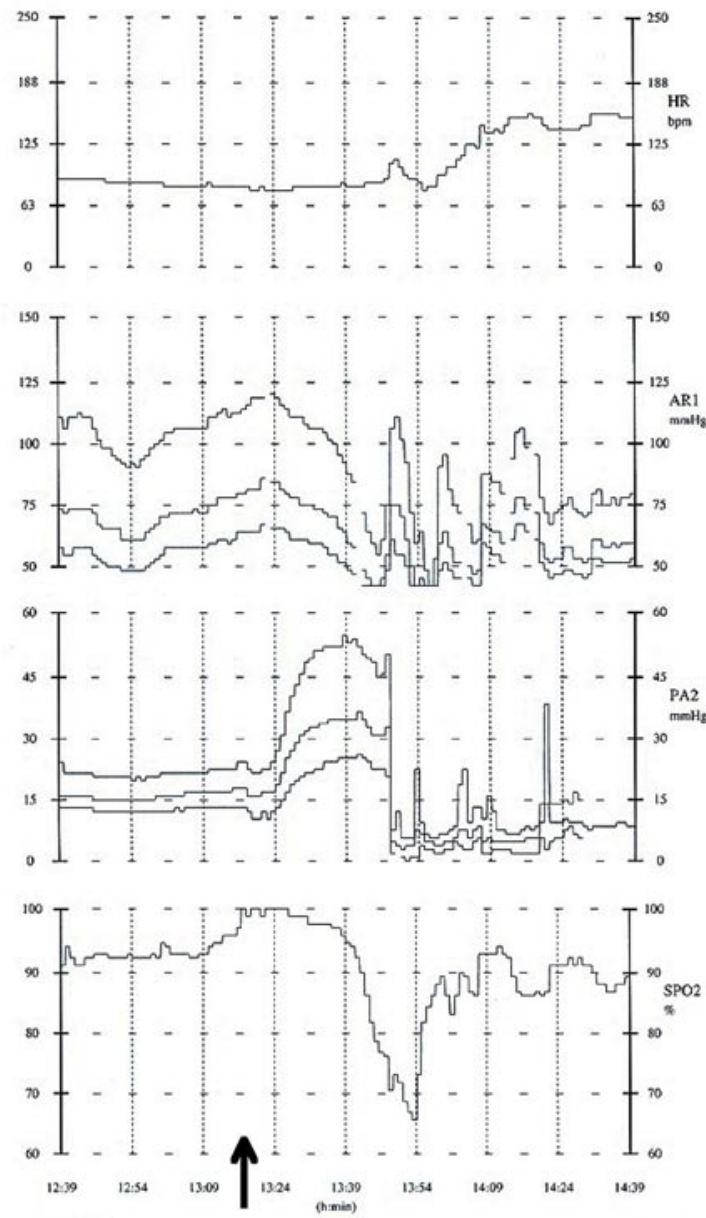

FIGURE 2 Graphic trend for heart rate (HR), arterial blood pressure (AR1), pulmonary artery pressure (PA2) and oxygen saturation by pulse oximetry $\left(\mathrm{SpO}_{2}\right)$. The black arrow indicates the time of completion of the platelet transfusion.

inhaled nitric oxide (see the Table for arterial blood gas measures). Hypotension was managed with aggressive fluid administration and infusions of epinephrine, norepinephrine and dopamine. Intravenous furosemide was administered twice; after each dose, the patient's urinary output increased but was accompanied by a decrease in blood pressure without improvement in oxygenation. Although an anaphylactic transfusion reaction was thought to have been unlikely, the patient also received intravenous diphenhydramine and hydrocortisone.

Leukocyte antibody investigations were sent to the Canadian Blood Services and antibody screening by granulocyte and lymphocyte immunofluorescence
TABLE Arterial blood gas measures

\begin{tabular}{lllll}
\hline Time & $12: 35$ & $14: 11$ & $14: 58$ & $16: 00$ \\
\hline FIO2 & 0.5 & 1.0 & 1.0 & 1.0 \\
pH & 7.41 & 7.24 & 7.20 & 7.35 \\
PO2 $(\mathrm{mmHg})$ & 93 & 75 & 53 & 83 \\
PCO2 $(\mathrm{mmHg})$ & 38 & 53 & 57 & 33 \\
Base excess & -1.3 & -5.3 & -7.5 & -6.8 \\
\hline
\end{tabular}

(GIFT, LIFT) using flow cytometry was performed at the St. Michael's Hospital Platelet Research Laboratory (Toronto, ON, Canada). A crossmatch of the plasma from the pooled platelet product against recipient leukocytes was positive on the LIFT and negative on the GIFT, interpreted as the presence of anti-human leukocyte antigen (HLA) antibody. On individual component crossmatch testing, plasma from two of the five whole blood derived platelet products demonstrated anti-HLA antibody and one demonstrated weak anti-HLA antibody. The donors were recalled and found to have anti-HLA antibody results concordant with component test results. Antibody specificity for HLA Class I was performed using Luminex (Tepnel Lifecodes Corporation, Stamford, CT, USA) by the University Health Network HLA Laboratory (Toronto, ON, Canada). The two donors who were crossmatch-positive for anti-HLA antibody by LIFT had multispecific Class I HLA antibodies and the donor with a weak anti-HLA antibody was negative for Class I HLA antibody.

Despite the severity of the patient's initial condition, his clinical course was ultimately favourable. There was minimal post-operative blood loss, with only $450 \mathrm{~mL}$ drained via chest tubes in the first $24 \mathrm{hr}$ after admission to the intensive care unit. He required no further blood transfusions. Within 48 hours of the TRALI event, inhaled nitric oxide was discontinued and ventilatory and hemodynamic support began to decrease. The radiographic findings resolved within days, and six days following the TRALI insult, the patient was weaned from the ventilator and his trachea was extubated. He was discharged from the hospital in good condition 14 days after surgery.

\section{Discussion}

Transfusion-related acute lung injury (ALI) was first identified as a clinical entity in 1983 when Popovsky ${ }^{2}$ described five patients who developed a clinical syndrome of respiratory distress, hypoxemia, hypotension, fever and bilateral pulmonary edema within four hours of transfusion. Since that time, an attempt has been made to formalize the definition of TRALI to facilitate 
diagnosis and reporting. The TRALI working group assembled by the National Heart, Lung and Blood Institute proposed a definition of TRALI as new ALI occurring during or within six hours of a transfusion in patients without pre-existing ALI before transfusion. ${ }^{3}$ The Canadian Consensus Conference separated TRALI into definite and possible TRALI, with the latter distinguished by the presence of risk factors for ALI. ${ }^{4}$ These risk factors included conditions causing direct lung injury (e.g., aspiration, pneumonia, toxic inhalation) and indirect lung injury (e.g., severe sepsis, shock, multiple trauma, acute pancreatitis, cardiopulmonary bypass). ALI was further defined based on the North American-European Consensus Conference ${ }^{5}$ as acute onset hypoxemia with a $\mathrm{PaO}_{2} / \mathrm{F}_{\mathrm{I}} \mathrm{O}_{2}$ ratio of less than $300 \mathrm{mmHg}$ or room air oxygen saturation of less than $90 \%$; bilateral infiltrates on chest radiograph; and the absence of circulatory overload or left atrial hypertension. In patients with other risk factors for ALI, TRALI can only be diagnosed after careful scrutiny of the patient's clinical course. Other TRALI-associated findings include fever, tachycardia, hypotension or hypertension, cyanosis and transient leukopenia. ${ }^{3,6}$ The case described above represents that of TRALI in a patient with a risk factor for ALI (cardiopulmonary bypass), but with a clear temporal course and a positive leukocyte antigen-antibody crossmatch confirming the diagnosis.

The pathophysiology of TRALI remains to be fully elucidated. However, there are two predominant hypotheses suggested, both of which result in increased permeability of the pulmonary capillary endothelium and non-cardiogenic, protein-rich pulmonary edema. The first is the antibody-antigen hypothesis ${ }^{7}$ wherein donor antibodies in the transfused component recognize cognate antigens on recipient leukocytes. As a result, recipient neutrophils are activated and sequestered within the pulmonary capillaries. Their subsequent degranulation induces pulmonary endothelial damage leading to capillary leak. In less than $10 \%$ of cases, pathogenic antibodies are present in the recipient with corresponding antigens on the donor leukocytes. ${ }^{8}$ In the neutrophil priming hypothesis, ${ }^{9}$ two events are required to cause TRALI. The first event is caused by the recipient's underlying clinical condition (surgery, infection or inflammation), which activates the pulmonary endothelium leading to sequestration of neutrophils in the pulmonary circulation. The second event involves transfusion of anti-leukocyte antibodies or biological response modifiers in stored blood components, which in turn activate the primed neutrophils resulting in pulmonary endothelial damage and capillary leak. The case described above showed the presence of multispecific anti-HLA Class I antibodies in two of the five platelet donors reacting in crossmatch testing against the patient's leukocytes, in the setting of post-cardiopulmonary bypass. In this case, TRALI likely resulted from a combination of patient factors and transfused donor antibody but the relative contribution of each factor cannot be determined.

Because the patient was under intensive continuous hemodynamic monitoring, this case provided a rare glimpse into the evolving vasomotor derangements that often accompany TRALI. ${ }^{10}$ In a case series of patients developing suspected TRALI post-liver transplant, ${ }^{11}$ PA pressures were obtained in four patients, with results ranging from $25 / 12$ to $41 / 30 \mathrm{mmHg}$. However, only single measurements were provided for each patient, and the timing of these measurements relative to the infusion of blood products was not reported. Brander et al. ${ }^{12}$ included a series of PA pressure measurements in their case report of a patient with TRALI, but the first measurement was not taken until two hours after the onset of hypoxemia. This showed moderate pulmonary hypertension of 33/20 $\mathrm{mmHg}$, which gradually returned to normal within the next few days.

The significance and mechanism of the altered pulmonary hemodynamics in this case is unclear. The onset of pulmonary hypertension was not consistent with either hypoxia-induced vasoconstriction or circulatory overload. It is possible that endothelial injury, thought to be a central component in the pathophysiology of TRALI, ${ }^{13}$ resulted in either formation of occlusive platelet thrombi or induction of smooth muscle constriction. The development of acute pulmonary leukostasis is not a mechanism supported by animal data. In fact these animal models suggest that pulmonary hypertension may only be a surrogate marker of TRALI with little pathophysiologic significance of its own. ${ }^{13,15,16}$ The subsequent development of pulmonary hypotension in this case appears to have been secondary to the patient's generalized cardiovascular collapse, a not infrequently reported occurrence in TRALI that likely reflects the development of a systemic inflammatory response syndrome.

The treatment for TRALI is supportive, and in severe cases may include positive pressure ventilation and inotrope infusions. Diuretics are not indicated, and the usefulness of steroids in TRALI, as with other forms of ALI, is still a matter of debate. A recent literature review concluded that inhaled nitric oxide is of little benefit in the treatment of ALI, ${ }^{17}$ and is unlikely to have had much impact on the case described, in which the patient had already developed pulmonary hypotension. Despite the paucity of effective interven- 
tions, the prognosis of TRALI is considerably better than with other forms of ALI. In a case series of 36 patients, ${ }^{7} 81 \%$ had a rapid, complete recovery within $96 \mathrm{hr}$. However, in $20 \%$ of cases, chest infiltrates and hypoxemia persisted longer than a week. Even with these favourable outcomes, mortality is still $5-10 \% .7,18$ According to data reported by the Canadian Blood Services at the Canadian Consensus Conference, mortality from TRALI was $17 \% .{ }^{19}$ Data from the Food and Drug Administration evaluating transfusion related fatalities from 2001 to $2003,{ }^{19}$ showed that TRALI had emerged as the leading cause of transfusion-related death accounting for $16.3 \%$ of transfusion deaths followed by $\mathrm{ABO}$ incompatibility and bacterial contamination.

As no specific treatment exists, focus must turn to prevention. It is essential for clinicians to report TRALI reactions to the blood bank so that appropriate investigations can be initiated to identify donors with antibodies who may potentially cause reactions in future recipients. Most blood centres have developed policies to address investigations and management of associated donors. ${ }^{20}$ Often this means the exclusion of implicated donors from future donation of high plasma-containing components, though TRALI has been described with the transfusion of blood products containing low volumes of plasma such as red blood cells and cryoprecipitate. ${ }^{6}$

As part of the preventive strategy, blood centres are also examining primary prevention policy changes to reduce TRALI. In the United Kingdom (UK), TRALI implicated blood products were $47 \%$ attributable to plasma-rich products including fresh frozen plasma, cryosupernatant and platelets even though these products represented only $25 \%$ of all blood products issued. ${ }^{19}$ As of 2003 , the National Blood Service in the UK moved to predominantly male plasma for transfusion, thereby minimizing plasmacontaining products from female donors at higher risk of developing leukocyte antibodies due to prior pregnancy. In November 2006, the AABB (formerly known as American Association of Blood Banks) published recommendations ${ }^{A}$ to minimize high-plasma volume blood component production from donors who are known to be leukocyte immunized or at high risk of leukocyte alloimmunization. In particular, this represents women with a history of pregnancy and donors with a history of transfusion. Though these measures address antibody-mediated TRALI, they do

\footnotetext{
A Strong M, Lipton KS. AABB Association Bulletin \#06-07.

Transfusion related acute lung injury.
}

not address TRALI that may result from other nonantibody mediated mechanisms such as biological response modifiers transfused to a susceptible patient.

Ultimately, the only measure that will reduce all possible cases of TRALI is to minimize unnecessary blood transfusions. In fact, this will not only reduce the risk of TRALI, but that of other transfusion adverse events, including $\mathrm{ABO}$ incompatibility, bacterial contamination and other transfusion-transmitted diseases. Whether platelets were indicated in this case is debatable, as there was only a moderate amount of blood loss when platelets were transfused. The decision, however, was likely influenced by the recent use of anti-platelet drugs and the expectation for ongoing bleeding. Nevertheless, this case illustrates that blood products have serious risks, and should only be transfused when absolutely necessary.

\section{Acknowledgements}

We thank Barbara Hannach for her critical review of the manuscript and K. W. Annie Bang and Neal denHollander for their input regarding the laboratory methods described.

\section{References}

1 Karkouti K, Wijeysundera DN, Yau TM, et al. Platelet transfusions are not associated with increased morbidity or mortality in cardiac sugery. Can J Anesth 2006; 53: 279-87.

2 Popovsky MA, Abel MD, Moore SB. Transfusion-related acute lung injury associated with passive transfer of antileukocyte antibodies. Am Rev Respir Dis 1983; 128: 185-9.

3 Toy P, Popovsky MA, Abraham E, et al.; National Heart, Lung and Blood Institute Working Group on TRALI. Transfusion-related acute lung injury: definition and review. Crit Care Med 2005; 33: 721-6.

4 Kleinman S, Caulfield T, Chan P, et al. Toward an understanding of transfusion-related acute lung injury: statement of a consensus panel. Transfusion 2004; 44: 1774-89.

5 Bernard GR, Artigas A, Brigham KL, et al. Report of the American-European consensus conference on acute respiratory distress syndrome: definitions, mechanisms, relevant outcomes, and clinical trial coordination. Consensus Committee. J Crit Care 1994; 9: 72-81.

6 Webert KE, Blajchman MA. Transfusion-related acute lung injury. Transfus Med Rev 2003; 17: 252-62.

7 Popovsky MA, Moore SB. Diagnostic and pathogenetic considerations in transfusion-related acute lung injury. Transfusion $1985 ; 25$ : 573-7.

8 Engelfriet CP, Reesink $H W$, Brand A, et al. Transfusion-related acute lung injury (TRALI). Vox 
Sang 2001; 81: 269-83.

9 Silliman CC. The two-event model of transfusionrelated acute lung injury. Crit Care Med 2006; 34(5 Suppl): S124-31.

10 Popovsky MA, Haley NR. Further characterization of transfusion-related acute lung injury: demographics, clinical and laboratory features, and morbidity. Immunohematol 2000; 16: 157-9.

11 Yost CS, Matthay MA, Gropper MA. Etiology of acute pulmonary edema during liver transplantation: a series of cases with analysis of the edema fluid. Chest 2001; 119: 219-23.

12 Brander L, Reil A, Bux J, Taleghani BM, Regli B, Takala J. Severe transfusion-related acute lung injury. Anesth Analg 2005; 101: 499-501.

13 Bux J, Sachs UJ. The pathogenesis of transfusion-related acute lung injury (TRALI). Br J Haematol 2007; 136: 788-99.

14 Seeger W, Schneider U, Kreusler B, et al. Reproduction of transfusion-related acute lung injury in an ex vivo lung model. Blood 1990; 76: 1438-44.

15 Silliman CC, Voelkel NF, Allard JD, et al. Plasma and lipids from stored packed red blood cells cause acute lung injury in an animal model. J Clin Invest 1998; 101: 458-67.

16 Silliman CC, Bjornsen AJ, Wyman TH, et al. Plasma and lipids from stored platelets cause acute lung injury in an animal model. Transfusion 2003; 43: 633-40.

17 Adhikari NK, Burns KE, Friedrich JO, Granton JT, Cook DJ, Meade MO. Effect of nitric oxide on oxygenation and mortality in acute lung injury: systematic review and meta-analysis. BMJ 2007; 334: 779.

18 Popovsky MA, Chaplin HC Jr, Moore SB. Transfusionrelated acute lung injury: a neglected, serious complication of hemotherapy. Transfusion 1992; 32: 589-92.

19 Goldman M, Webert KE, Arnold DM, Freedman J, Hannon J, Blajchman MA; TRALI Consensus Panel. Proceedings of a consensus conference: towards an understanding of TRALI. Transfus Med Rev 2005; 19: 2-31.

20 Wendel S, Biagini S, Trigo F, et al. Measures to prevent TRALI. Vox Sang 2007; 92: 258-77. 